\title{
Uncertainty Handling in Navigation Services using Rough and Fuzzy Set Theory
}

\author{
Anahid Basiri \\ Dept. Computer Science, \\ National University of Ireland \\ Maynooth, Ireland \\ Anahid.basiri@nuim.ie \\ Pouria Amirian \\ Dept. Computer Science, \\ National University of Ireland Maynooth, \\ Ireland \\ Amirian@cs.nuim.ie
}

\author{
Adam Winstanley \\ Dept. Computer Science, \\ National University of Ireland Maynooth, \\ Ireland \\ adam.winstanley@cs.nuim.ie \\ Colin Kuntzsch \\ Institute of Cartography and \\ Geoinformatics, Leibniz University, \\ Hannover, Germany \\ Colin.Kuntzsch@ikg.uni-hannover.de
}

\author{
Monika Sester \\ Institute of Cartography and \\ Geoinformatics, Leibniz University, \\ Hannover, Germany \\ Monika.Sester@ikg.uni-hannover.de
}

\begin{abstract}
Navigation services, such as used in cars, are widely used nowadays. Many applications, positioning technologies and techniques have been developed to make navigation systems easier to use. However current navigation systems suffer from different aspects of uncertainty such as incomplete or inaccurate positional data. This paper reviews aspects of uncertainty which should be considered when developing navigation systems. A proposed approach, based on rough set and fuzzy set theories, is explained and implemented in an application.
\end{abstract}

\section{Categories and Subject Descriptors}

I.2.3 [Deduction and Theorem Proving]: Uncertainty, "fuzzy," and probabilistic reasoning

\section{General Terms}

Theory

\section{Keywords}

Navigation Services; Uncertainty; Rough Set Theory; Fuzzy Set Theory; Location Based Services; Spatio-Temporal Objects

\section{INTRODUCTION}

Location Based Services (LBS) are now widely used by mobile users for many tasks, such as finding the current location on the map or listing the location of nearby shops or facilities. An important category of LBS is navigation services which mainly provide users with instructions to travel to their required destination from their current location using the best route calculated from a combination of user preferences and the underlying road network. Mobile devices mostly employ the Global Positioning System (GPS) to provide location. Although GPS is one of the most widely used technologies for this, sometimes it does not provide location with an acceptable degree of accuracy due to attenuation, fading and multipath problems. This inaccuracy is one of the most important aspects of uncertainty

Permission to make digital or hard copies of all or part of this work for personal or classroom use is granted without fee provided that copies are not made or distributed for profit or commercial advantage and that copies bear this notice and the full citation on the first page. To copy otherwise, or republish, to post on servers or to redistribute to lists, requires prior specific permission and/or a fee.

ACM SIGSPATIAL QUeST'12, November 6, 2012. Redondo Beach, CA, USA,Copyright (c) 2012 ACM ISBN 978-1-4503-1700-9/12/11..\$15.00 in navigation.

Uncertainty may be classified according to the following aspects [1]: inaccuracy and error, vagueness, incompleteness, inconsistency and imprecision. Positional inaccuracy can of course be considered to fall under inaccuracy and error. However, from another point of view, GPS data may suffer from incompleteness also because GPS can only provide accurate location where there is an unobstructed line of sight to at least four satellites. Another category, vagueness, occurs within navigational instruction provided to users. In current navigation systems, the navigational instructions are provided to user as they approach a turning point. So navigational instructions are updated and become available "close to" each turning point. Deciding when the instruction should be given to the user is a complicated decision since "approaching" and "close to" do not have mathematically and spatially clear definitions.

Uncertainty, as defined above, is not limited to these examples. Section two focuses on aspects of uncertainty through further examples. In order to handle this uncertainty, we propose using a fuzzy inference system to handle vagueness in the definition of the spatial area in which navigational instructions should get updated. Fuzzy set theory was originally developed to solve the problem of vagueness in definitions and variables and is one of the most widely used techniques for handling uncertainty. Applications, systems and services in many domains have been developed based on fuzzy set theory [2], [3], [4]. While the performance of fuzzy inference systems is still under question [5], there has been some research regarding their optimization [6], [7]. However, due to the complicated mathematical background of fuzzy set theory [8], its computational overhead is not usually appropriate for real time navigational purposes. Rough set theory [9] is one of the simplest uncertainty handling frameworks and is proposed here to deal with aspects of uncertainty in navigation systems. An introduction to the principles of rough set theory and fuzzy set theory is given briefly in section three. Then section four implements the proposed model into an navigational application.

\section{UNCERTAINTY IN NAVIGATION SYSTEMS}

Generally speaking, almost all the information that we possess about the real world is uncertain, incomplete and imprecise. This section is focused on explaining the main aspects of uncertainty and how they are manifested in navigation services: inaccuracy 
and error, vagueness, incompleteness, inconsistency and imprecision.

\subsection{Inaccuracy and Error}

Inaccuracy and error refers to the deviations of captured or measured data from true values. Accuracy is the closeness of agreement between measured values and the true values. Error is the difference between a measurement and the true value of the quantity being measured. Since positioning is one of the most essential components of any navigational system, this part is mostly dedicated to locational accuracy rather than other measurements, such as attribute accuracy. Mobile devices mostly employ Global Positioning System (GPS) receivers to get positioning data. Factors that may affect the accuracy of GPS data are explained below. Since GPS is not only positioning technology implemented by navigation systems, a brief overview of other position technology is provided too.

Accuracy of positional data provided by GPS, embedded in mobile phones, may be affected by several different factors [10] such as signal multipath, availability of satellites, orbit geometry and also the quality of the GPS receiver [11]. The most important error is caused by multipath which can be introduced when signals are reflected by the façade of an object such as a building. As the less direct path will be longer and take extra time, this can add errors. Another important factor in GPS accuracy is the number visible satellites. Obviously the more satellites that can be seen and can be used to provide data, the more triangulation points are obtained and the greater the level of accuracy is achieved. The geometry of the satellite positions can have an impact on GPS errors. The optimum situation occurs when the satellites are observed at wide angles relative to each other. Poorer accuracy is obtained when the observed satellites are closer together.

There are other positioning technologies that can provide locations with differing degrees of accuracy. For example, mobile phone networks can determine the location of an individual within a suburb or town (around 150 to 300-m accuracy. The accuracy of the Enhanced Observed Time Difference (E-OTD) technique can be expected to be around $125 \mathrm{~m}$. One of the most widely used positioning technologies, which can be used seamlessly indoors and outdoors, uses Wireless Local Area Networks (WLAN). There are many factors which may affect accuracy of this technique that are related to the mobile device such its device scan interval. For an average error of 2 meters, the scan interval needs to be smaller than 2500 milliseconds.

Another solution for indoor positioning is using measurements provided by internal accelerometers and gyroscopes to model an Inertial Positioning System. The position and orientation of an object relative to a known starting point, orientation and velocity are calculated. The main challenge in inertial positioning is integration drift. That means small errors in the measurement of acceleration and angular velocity are integrated into progressively larger errors in velocity, which are compounded into still greater errors in position.

Bluetooth networks can also calculate the position of a device. Accuracy in a Bluetooth positioning system is dependent on the maximum range of the system like all wireless positioning techniques. The longer the range the higher the error can be. Bluetooth has a typical range from $10 \mathrm{~m}$ to $100 \mathrm{~m}$ depending on the power class of the device.
In summary, the various positioning technologies and techniques which have been implemented in navigation applications provide locational data to differing but estimable degrees of accuracy.

\subsection{Vagueness}

Another aspect of uncertainty in navigation systems is vagueness. Vagueness is related to the imprecision in concepts which are used for explaining phenomena. In current navigation systems, instructions are provided to the user turn-by-turn. So navigational instructions are getting updated and become available for users "close to" each turning point. Modeling and storing "close to" areas for each turning point, or node, would be another challenge since there has not been a predefined definition for "close to". It may cover a large extent that is distributed continuously in space and has indeterminate boundaries [12]. Such vague phrases as far, near and around must be defined mathematically and modeled spatially. This paper proposes fuzzy set theory [8], as one of the most powerful and widely used frameworks in uncertainty handling, to model such a vague concept. Fuzzy logic is originally developed to deal with linguistic variables. By a linguistic variable we mean a variable whose values are words or sentences in a natural or artificial language [13]. For example, distance is a linguistic variable if its values is linguistic rather than numerical, as in near, very near, quite near, far, not very far, and so on. Since the area in which navigational instructions get updated is defined by such linguistic variables, fuzzy set theory should be able to model this aspect of uncertainty.

\subsection{Incompleteness}

Incompleteness refers to a lack of relevant information. Each positioning technology may not provide locational data in all situations. For example GPS is mainly an outdoor positioning technology; consequently, it cannot provide the users' position while they are inside a building or a tunnel. Even outdoors there are many places where GPS signals simply are not available due to obstruction. For example, in the inner city streets of urban areas lined with skyscrapers the 'visibility' of GPS satellites is very limited. In such areas the signals can be either obstructed for extended periods of time or even continuously unavailable.

Generally speaking, most positioning technologies are not universal solutions for seamless location. For example, Bluetooth or RFID (Radio Frequency Identification) are reliant on network availability. However, it may be possible to use interpolation or extrapolation techniques [14] to estimate position when the technology cannot provide any data. There are many solutions to position estimation, but it is outside the scope of this paper to discuss these.

\subsection{Inconsistency}

Inconsistency refers to the conflicts between two or more sets of information or data describing the same variable. For example, if two sensors sense the same variable, such as temperature or pressure, it is possible to have two different values for the same variable at the same time. In current navigation services, usually this aspect of uncertainty has less priority since the most important variable to be measured is position and usually only one positioning technology is embedded in the device. However, more sophisticated devices have more than one positioning technology, for example for working seamlessly indoors and outdoors. This may result in inconsistent data being returned from sensors due to their differing accuracy.

Even when only one positioning technology is included, it is possible to encounter problems with inconsistent data. For 
example, if we are using a camera network as a positioning solution, it is possible to get two or more different locations calculated since a mobile object can be in view by more than one camera. In camera-based positioning technology, a set of cameras is to provide synchronized image data which is processed and exteriorly oriented to track or calculate the position of objects in those images. In order to get the position of a user within an image, using image processing techniques objects are detected and localized to a particular cell. Then the absolute or relative position of that cell is calculated. In order to follow the user continuously, there should be an overlapping area between each pair of cameras. This overlapping area allows the system to follow users from one camera's area of coverage to another one. If a mobile user is located in the overlapping area viewed by two or more cameras, each camera will calculate a position for that user. The position may differ if the resolution and configuration of cameras differ. Consequently, each camera may calculate a position which is different resulting in inconsistent positional data.

\section{SOLUTION}

As demonstrated above, location based services suffer from different aspects of uncertainty. Many solutions have been proposed including possibility theory [15] and Dempster-Shafer evidence theory [16]. Rough set theory, introduced by Pawlak [9], represents the uncertainty of an event by the approximation of a collection of sets. Rough set theory is one of the most powerful devices to deal with uncertainty while, in comparison with other theories, it is very simple to implement which makes it very attractive for location based services where time and performance are important criteria. Most of the time, location based services are provided to moving users whose positions can change rapidly. Consequently, it is very important to provide them with the services as fast as possible to be relevant to the current location. Rough set theory has one of the simplest frameworks, so its use could be appropriate.

In order to handle vagueness it is better to implement fuzzy set theory which essentially has been developed to handle this aspect of uncertainty and is one of the most powerful and widely used methods. Many fuzzy inferences have been developed and can be used to define which area should be considered as "around" or "near" to a spatial feature [18]. In the following, we briefly explain the principles of rough and fuzzy set theory.

\subsection{Rough Set Theory}

Suppose some uncertain phenomenon $\mathrm{X}$ is approximated using only the information contained in a set B. We can construct the B-

lower and B-upper approximations of $\mathrm{X}$, denoted by $\frac{B X}{\text { and }} \overline{B X}$ respectively [9]. The lower approximation of $X$ is the collection of objects which can be classified with full certainty as members of the set $\mathrm{X}$. The upper approximation of $\mathrm{X}$ is the collection of objects that may possibly be classified as members of the set $\mathrm{X}$. The set $B N_{B}(X)=\overline{B X}-\underline{B X}$ is called the B-boundary region of $X$, and thus consists of those objects that we cannot classify with certainty to be either inside $\mathrm{X}$, or outside $\mathrm{X}$.

As it explained in next sub section, rough set theory is implemented to handle uncertainty due to inaccuracy and incompleteness of positional data in location based services. In order to handle vagueness of linguistic variable used in location based services, a fuzzy inference system is developed. Following is a brief explanation regarding to fuzzy set theory and fuzzy inference systems.

\subsection{Rough Set Based Positioning}

Measurement of location suffers from inaccuracy, incompleteness and inconsistency. The simplicity of rough set theory enables us to handle uncertainty of positional data without compromising performance due to the computational overhead. Since users' positions change continuously, it is very important to use a framework which handles uncertainty with an acceptable level of performance. In this regard, rough set theory is considered as one of the simplest uncertainty handling framework.

Since rough set theory uses three main regions to interpret a spatial feature [5], this paper proposes considering a three ring polygon to model a position. Around the position of the user, typically a latitude and a longitude, there are two buffer rings. The radii of these buffers model the level of accuracy of the positional data with greater accuracy meaning smaller buffers. In addition, depending on the speed and direction of movement, the buffer feature exhibits deformation in the direction of movement (figure 1). The smaller buffer around the point feature defines the area in which there is a high likelihood of the user being located. This area corresponds to the lower-bound region from rough set theory. The larger buffer contains the area in which the user is located with less likelihood. Outside this, there is no possibility that it contains the true position.
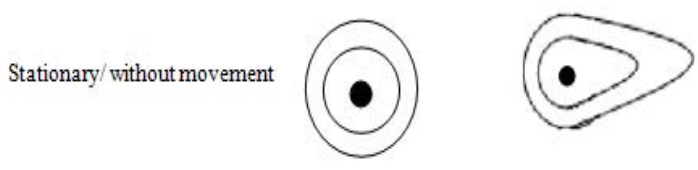

Moving

\section{Figure 1. Stationary and moving object positional area}

In following subsection a review of fuzzy set theory and fuzzy inference systems are provided, since they can be used to implement vagueness in some LBS related concepts.

\subsection{Fuzzy Set Theory}

Fuzzy logic starts with the concept of a fuzzy set. A fuzzy set can contain elements with only a partial degree of membership. Fuzzy set theory uses a continuous assessment of elements in a set from certain absence to certain membership; this is described with the aid of a membership function valued in the real unit interval $[0,1]$.

A fuzzy set is a pair $(\mathrm{A}, \mathrm{m})$ containing a set $\mathrm{A}$ and membership function $\mathrm{m}: \mathrm{A} \rightarrow[0,1]$ [8].The membership function of a fuzzy set is a generalization of the indicator function in classical sets [8]. A' the membership degree quantifies the grade of membership of the element $\mathrm{x}$ to the fuzzy set [8]. The value 0 means that $\mathrm{x}$ is not a member of the fuzzy set; the values between 0 and 1 characterize fuzzy members, which belong only partially.

Fuzzy set theory has been implemented to deduce which area is considered to be "around" an object by [18]. It is possible to extend this to model vagueness resulting by using this and other similar linguistic variables.

With both the model for positional uncertainty of a mobile user and definitions of vague terms such as "around", it is possible to determine with more rigor when a user has entered the area of "around" an object. This can be done using topological relationship validation [18]. A simple calculation can combine the 2D likelihoods surfaces to, for example, trigger the issuing of 
navigational instructions when a vehicle is approaching a road junction.

\section{CONCLUSION}

Location based services suffer from different aspects of uncertainty. Since there can be uncertainty in position of the user, incompleteness in positional data and vague definitions of spatial relationships, we should model this uncertainty. This paper focused on modeling uncertainty of location for LBS. Positional uncertainty was handled by rough set theory and vagueness was handled by fuzzy set theory.

\section{Acknowledgment}

Research presented in this paper was funded by a Strategic Research Cluster grant (07/SRC/I1168) by Science Foundation Ireland under the National Development Plan. The authors gratefully acknowledge this support.

\section{REFERENCES}

[1] Worboys M.F. 1998. Imprecision in finite resolution spatial data. GeoInformatica. 2(3): 257-279.

[2] Arabacioglu, B. C. 2010. Using fuzzy inference system for architectural space analysis. Applied Soft Computing 10 (3): 926937.

[3] Tsitolovsky L., Sandler U. 2008. Neural Cell Behavior and Fuzzy Logic. Springer. ISBN ISBN 978-0-387-09542-4.

[4] Pedrycz, W., Gomide, F. 2007. Fuzzy systems engineering: Toward Human-Centerd Computing. Hoboken: WileyInterscience. ISBN 978-0-471-78857-7.

[5]Homnan, B. 2004. The performance analysis for fuzzy inference system-based adaptive soft handoff thresholds. TENCON 2004. 2004 IEEE Region 10 Conference. 533 - 536 Vol. 2.

[6] Ramirez, E.M., Mayorga, R.V. 2008. On the Parameter Optimization of Fuzzy Inference Systems. International Journal of Information and Mathematical Sciences. 4:1.

[7] Tan, K., Tokinaga, S. 1999. Optimization of Fuzzy Inference Rules by Using the Genetic Algorithm and its Application to the Bond Rating. Journal of Operations Society of Japan. Vol. 2. No 3 .

[8]Zadeh, L.A. 1965. Fuzzy sets, Information and Control. 8338 353.

[9] Pawlak, Z., Polkowski, L. 1991. Rough set: A tutorial.

[10] Bowditch, N. 2002. The American Practical NavigatorChapter 11 Satellite Navigation. United States government.

[11] Rip, M.R., Hasik, J.M. 2008. The Precision Revolution: GPS and the Future of Aerial Warfare. Naval Press. ISBN 1-55750973-5.

[12] Bassiri, A., Malek, M.R., Amirian, P. 2012, Ambient shopping advertisement using rough service domain. Journal of Ambient Intelligence and Smart Environment, JAISE 4(2): 95-105.

[13] Zadeh, L.A. 1975. The concept of a linguistic variable and its application to approximate reasoning. Information Sciences, Vol. 8, No 3. 199-249.

[14] O'Hara, C., 2006, GPS SATELLITE SIGNAL LOCK LOSS PROBABILITY, Collection Mémoires et thèses électroniques, Laval Université, Canada.
[15] Dubois, D., Prade, H., 1988, Possibility Theory, New York: Plenum

[16] Shafer, G. 1976, A Mathematical Theory of Evidence. Princeton University Press, Princeton, New Jersey, USA.

[17] Sugeno, M., 1977, Fuzzy measures and fuzzy integrals: a survey, (M.M. Gupta, G. N. Saridis, and B.R. Gaines, editors) Fuzzy Automata and Decision Processes, pp. 89-102, NorthHolland, New York.

[18] Bassiri, A., Malek, M.R., Alesheikh, 2008, Rough patiotemporal Topological Relationships, Computational Intelligence in Decision and Control, published by World Scientific Publisher.

[19] Kosko B. 1992, Neural Networks and Fuzzy Systems--A Dynamic Systems Approach to Machine Intelligence. PrenticeHall, Englewood Cliffs. 